\title{
Curcumin inhibits human cytomegalovirus by downregulating heat shock protein 90
}

\author{
YALI LV ${ }^{1}$, LILI GONG ${ }^{1}$, ZIHUI WANG ${ }^{1}$, FEIFEI HAN ${ }^{1}$, HE LIU $^{1}$, XUECHUN LU ${ }^{2}$ and LIHONG LIU ${ }^{1}$ \\ ${ }^{1}$ Beijing Chao-Yang Hospital, Capital Medical University, Beijing 100020; \\ ${ }^{2}$ Department of Geriatric Hematology, PLA General Hospital, Beijing 100853, P.R. China
}

Received September 30, 2014; Accepted June 11, 2015

DOI: $10.3892 / \mathrm{mmr} .2015 .3983$

\begin{abstract}
Curcumin is a traditional Chinese medicine extracted from the rhizome of the herb Curcuma longa, which exhibits anti-human cytomegalovirus (HCMV) activity, however, the underlying mechanism remains to be elucidated. The present study reported that the pharmacogenomics of curcumin are similar to that of the antiviral drug, geldanamycin, which targets heat shock protein 90 (Hsp90). Comparative analysis of 3,000 clinical drugs demonstrated that curcumin had a positive association with the gene expression profiles of several drugs, among which the pharmacogenomics of the antiviral drug, geldanamycin, were most similar to that of curcumin. Molecular docking simulation analysis revealed that curcumin fit well in the binding pocket of Hsp90, with hydrogen bonds, hydrophobic interactions and conjugation to maintain adhesion. Consistently, HCMV infection of human embryonic lung fibroblast cells resulted in increased expression of Hsp90 $\alpha$, which was significantly inhibited by treatment with curcumin. These findings suggested that targeting Hsp90 contributed to the anti-HCMV activity of curcumin.
\end{abstract}

\section{Introduction}

Human cytomegalovirus (HCMV), a member of the herpes virus family, is the leading viral cause of congenital abnormalities and mental impairment in newborns, and contributes significantly to morbidity and mortality rates in immunocompromised persons. HCMV can be transmitted via multiple pathways, including saliva, sexual contact, placental transfer, breast-feeding and blood transfusion (1). Over one-third of transplant recipients are infected with $\mathrm{HCMV}$, and the mortality rate of these patients is as high as $85 \%$, and $40 \%$ of patients with acquired immunodeficiency syndrome, who have a secondary

Correspondence to: Dr Lihong Liu, Beijing Chao-Yang Hospital, Capital Medical University, 8 Gongren Tiyuchang South Road, Beijing 100020, P.R. China

E-mail: lvyali_happy@163.com

Key words: curcumin, geldanamycin, pharmacogenomics, heat shock protein 90 , bioinformatics infection of HCMV exhibit retinopathy, gastrointestinal damage and central nervous system disorders $(2,3)$. In addition, latent HCMV infection is closely associated with cardiovascular disease, stroke, diabetes and other chronic diseases $(4,5)$. However, the cellular and molecular mechanisms of the persistent infection, latency and reactivation of HCMV remain to be fully elucidated (2). Following the widespread introduction of anti-HCMV drugs, including ganciclovir, valganciclovir and foscarnet, there has been a significant reduction in the number of HCMV infections, however, these drugs have been demonstrated to cause potentially serious side effects, exhibit low bioavailability and the inability to eliminate carrier states, and issues with drug resistance, patient compliance and cost, further limiting their effectiveness and wide application (3). Therefore, the development of safe and effective anti-HCMV drugs with novel mechanisms of action is urgently required.

Curcumin is a traditional Chinese medicine, which is extracted from the rhizome of the herb Curcuma longa (6). Curcumin has a low toxicity and has been widely used for the prevention of viral infection and other diseases, including cancer (7-9). Our previous study demonstrated that curcumin has significant anti-HCMV activity and improves the survival rate of host cells by inhibiting the HCMV DNA replication, apoptosis and inflammation induced by HCMV infection (10). However, the mechanism of anti-HCMV activity of curcumin remains to be fully elucidated.

In the development of novel drugs, bioinformatics is capable of identifying the key molecular targets in the pathological process and confirming the association between the structure and function of the drug. Bioinformatics has now become an important biopharmaceutical tool, which reduces the requirement for manpower and resources. The widespread application of bioinformatics has significantly accelerated the understanding and the development of novel drugs for several types of disease (11). Our previous studies have successfully applied bioinformatics to screen drugs for the treatment of leukemia (12) and aplastic anemia (13). In the present study, the anti-HCMV mechanism of curcumin was determined using bioinformatics, which was further confirmed by subsequent experiments.

\section{Materials and methods}

Reagents and kits. Dulbecco's modified Eagle's medium (DMEM), fetal bovine serum (FBS), SYBR Green, 
penicillin, streptomycin, L-glutamine, trypsin, dimethyl sulfoxide (DMSO), Triton X-100, phenylmethanesulfonyl fluoride (PMSF) and $\beta$-mercaptoethanol were obtained from Sigma-Aldrich (St. Louis, MO, USA). Rabbit anti-heat shock protein 90 (Hsp90) antibody was purchased from Cell Signaling Technology (Boston, MA, USA). Goat anti-rabbit secondary antibody was obtained from Invitrogen Life Technologies (Carlsbad, CA, USA).

Cell culture and virus propagation. Human embryonic lung fibroblast (HELF) cells were purchased from the National Platform of Experimental Cell Resources for Sci-Tech (Beijing, China) and were cultured in DMEM, supplemented with $10 \% \mathrm{FBS}, 100 \mathrm{U} / \mathrm{m}$ penicillin, $100 \mu \mathrm{g} / \mathrm{ml}$ streptomycin, and $2 \mathrm{mM} \mathrm{L}$-glutamine at $37^{\circ} \mathrm{C}$ in a humidified atmosphere of $95 \%$ air and $5 \% \mathrm{CO}_{2}$. The HCMV laboratory strain, AD169, was purchased from American Type Culture Collection (Manassas, VA, USA) and prepared in the HELF cells maintained in DMEM, containing 2\% FBS (maintenance medium).

Antiviral compounds. Curcumin was obtained from the National Institute for Food and Drug Control (Beijing, China) and stored as a $50 \mathrm{mM}$ stock solution in DMSO for in vitro use. The high, middle and low doses of curcumin used in the present study were 2,1 and $0.5 \mu \mathrm{M}$, respectively. Intravenous formulations of $0.2 \mathrm{mM}$ ganciclovir (GCV; Sigma-Aldrich) and $0.5 \mu \mathrm{M}$ geldanamycin (Sigma-Aldrich) in DMSO were used as references.

Drug genome similarity analysis of transcription. The mechanism of action of curcumin was predicted from pharmacogenomics databases, using bioinformatics to perform genome-wide transcriptional analysis of similarity. Using curcumin as a keyword, associated gene expression information was identified in various gene expression databases, including Gene Expression Omnious, BodyMap (Human and Mouse gene expression database), ArrayExpress Database (Public repository for microarray-based gene expression data), RNA Abundance Database, Stanford Microarray Database, Human Gene Expression and ChipDB, a searchable database of gene expression. These databases are open access database resources and can be accessed online. According to the data in the GenBank and internet platforms, the cloned drug genome sequences of curcumin were transferred to the above online databases to determine the expression information.

Molecular docking simulation. ChemBio Office (2010) was used to draw the molecular structure of curcumin, and the resultant files were saved in the standard delay format. MOE (2010) was applied to analyze the three-dimensional minimum energy conformations. The docking parameters in MOE were set as follows: The placement method, the first scoring function rescoring 1 and the saved poses were Triangle Matcher, London $\mathrm{dG}$ and 30, respectively. In addition, the refinement, the second refinement scoring function rescoring 2 and the saved poses were set to force field, none and 10, respectively (7).

Western blotting. The HELF cells were seeded at a density of $1 \times 10^{6} / \mathrm{ml}$ in tissue culture flasks and cultured for $12 \mathrm{~h}$. The cells were subsequently infected with HCMV at 100 times the
Table I. Curcumin pharmacogenomics to analyze the similarity of transcription.

\begin{tabular}{lc}
\hline Drug & $\begin{array}{c}\text { Number of similar gene } \\
\text { expression profiles }\end{array}$ \\
\hline Geldanamycin & 10 \\
Astemizole & 3 \\
Mefloquine & 3 \\
Thioridazine & 8 \\
Withaferin A & 2 \\
Vorinostat & 9 \\
\hline
\end{tabular}

median tissue culture infective dose $/ 0.1 \mathrm{ml}$. Following incubation at $37^{\circ} \mathrm{C}$ for $2 \mathrm{~h}$ under a humidified atmosphere of $95 \%$ air and $5 \% \mathrm{CO}_{2}$, the supernatant was removed and replaced with maintenance medium, with or without curcumin. Following an additional $48 \mathrm{~h}$ at $37^{\circ} \mathrm{C}$, the cells were harvested and washed twice with cold phosphate-buffered saline and lysed in cold radioimmunoprecipitation buffer, containing $25 \mathrm{mM}$ Tris- $\mathrm{HCl}$ (pH 7.6), $150 \mathrm{mM} \mathrm{NaCl}, 1 \% \mathrm{NP}-40,1 \%$ sodium deoxycholate, $1 \mathrm{mM}$ PMSF and $0.1 \%$ sodium dodecyl sulfate (SDS), on ice for $30 \mathrm{~min}$, followed by centrifugation at $9,600 \mathrm{x} \mathrm{g}$ at $4^{\circ} \mathrm{C}$ for $10 \mathrm{~min}$. Protein quantification was performed using a bicinchoninic acid protein assay (Pierce, Rockford, IL, USA). Equal quantities of protein $(50 \mu \mathrm{g})$ from the different groups were denatured in SDS sample buffer and separated by $8 \%$ SDS-polyacrylamide gel electrophoresis. The proteins were transferred onto polyvinylidene difluoride membranes in buffer, containing $25 \mathrm{mM}$ Tris, $192 \mathrm{mM}$ glycine and $20 \%$ methanol. The membranes were blocked with Tris-buffered saline, containing TBS; $137 \mathrm{mM}$ $\mathrm{NaCl}$ and $20 \mathrm{mM}$ Tris- $\mathrm{HCl}$ (pH 7.5), containing $0.1 \%$ Tween 20 and $5 \%$ dried non-fat milk powder at room temperature for $2 \mathrm{~h}$. Following blocking, the blots were incubated with primary antibodies against rabbit anti-human Hsp 90 (mAb; Cell Signaling Technologies, Danvers, MA, USA; cat. no. 4877) and mouse anti-human $\beta$-actin (mAb, ZSJB-Bio, Beijing, China; cat. no. 131023) at 1:1,000 in TBS containing $0.05 \%$ Tween-20 (TBST) at $4^{\circ} \mathrm{C}$ overnight. The membranes were subsequently washed with TBST three times. The blots were then incubated with Alexa Fluor ${ }^{\circledR} 680$ goat anti-mouse IgG and goat antirabbit $\mathrm{IgG}$ at 1:10,000 (Invitrogen Life Technologies, Grand Island, NY, USA) in TBST. After washing the membranes with TBS, the signals were detected using Odyssey infrared laser imaging system (LI-COR Biosciences, Inc,. Lincoln, NE, USA), according to the manufacturer's instructions. For densitometric analysis of western blotting images, density profiles of the bands were measured using ImageJ software (National Institute of Health, Bethesda, MA, USA).

Statistical analysis. The data were analyzed using Microsoft Office Excel 2003 (Microsoft, Redmond, WA, USA) and expressed as the mean \pm standard error of the mean. Group comparisons were evaluated using one-way analysis of variance, and significant differences between treatments were determined using the post-hoc test with Tukey-Kramer's honestly significant difference simultaneous pairwise mean comparison. $\mathrm{P}<0.05$ was considered to indicate a statistically significant difference. 


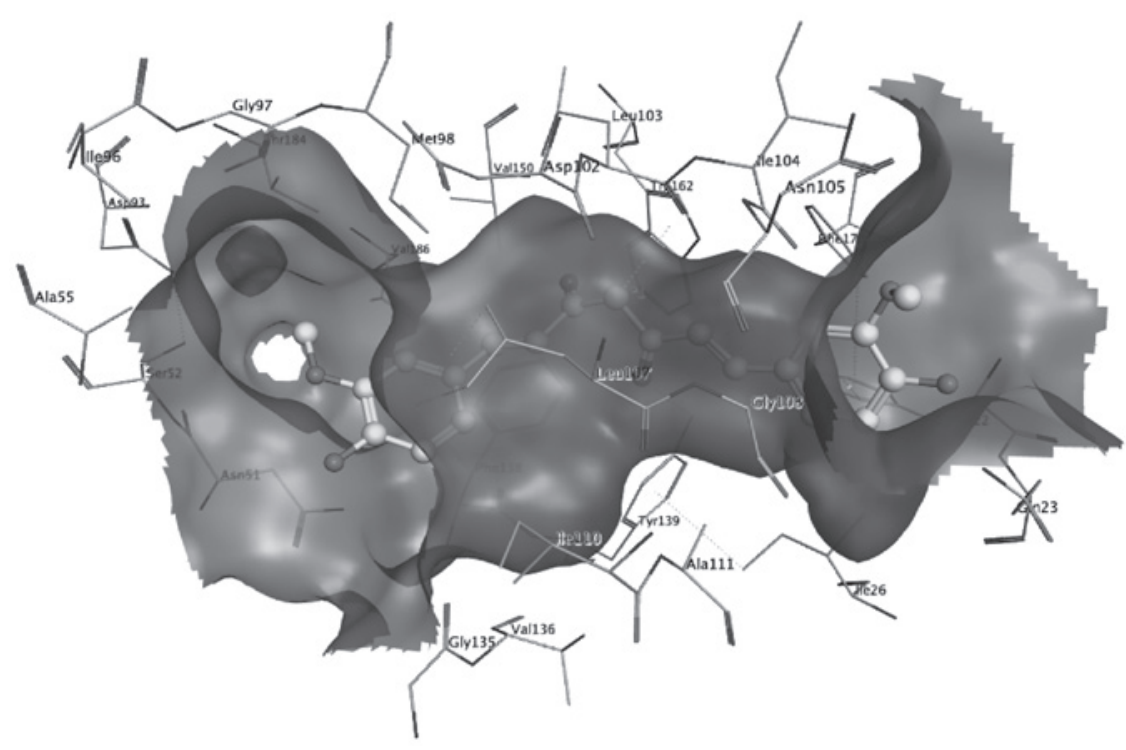

Figure 1. Curcumin fits well into the binding pocket of Hsp90. Molecular docking simulation of curcumin with Hsp90 revealed that hydrogen bonds, hydrophobic interactions and conjugation maintained the predominant driving force of adhesion. Hsp90, heat shock protein 90.

\section{Results}

Curcumin genome similarity analysis of transcription. To investigate the pharmacogenomics of curcumin, a comparative analysis of 3,000 clinical drugs was performed, which revealed that the gene expression profiles of curcumin had a positive association with several drugs (Table I). Among these drugs, the pharmacogenomics of curcumin were most similar to that of the antiviral drug, geldanamycin. Geldanamycin exerts an antiviral effect, predominantly by specifically binding to Hsp90 (14). This analysis suggested that curcumin may inhibit HCMV by targeting Hsp90.

Molecular docking simulation of curcumin with Hsp90. To confirm the above hypothesis, a molecular docking simulation of curcumin with Hsp90 was performed, which revealed that curcumin fit well into the binding pocket of Hsp90, among which hydrogen bonds, hydrophobic interactions and conjugation maintained the predominant driving force of adhesion (Fig. 1). As shown in Fig. 2, a carbonyl oxygen atom of curcumin acted as a hydrogen bond acceptor to form a hydrogen bond with Tyr139 of Hsp90. In addition, Trp162 and Phe22 of Hsp90 were conjugated with curcumin (Fig. 2). Furthermore, Ile26, Leu107, Ile104, Phe170, Leu103, Ala111 and other hydrophobic amino acids of Hsp90 formed hydrophobic interactions with curcumin (Fig. 2).

Curcumin reduces the protein expression of $H s p 90 \alpha$. The present study investigated the effect of curcumin on the protein expression of Hsp90 $\alpha$ using western blotting. In contrast to the mock-treated cells, infection with HCMV in the curcumin-treated cells led to a significant increase in the protein expression of $\mathrm{Hsp} 90 \alpha$ (Fig. 3; P<0.05). As expected, treatment with geldanamycin, but not GCV, markedly eliminated the induction of the protein expression of Hsp90 $\alpha$ by HCMV. Notably, curcumin also inhibited the upregulated protein expression of Hsp90 $\alpha$ by HCMV in a dose-dependent manner (Fig. 3).

\section{Discussion}

In the present study, it was demonstrated that the pharmacogenomics of curcumin were most similar to that of geldanamycin, which is an inhibitor of Hsp90 and exerts a wide-spectrum antiviral role (15). The in vitro experiments revealed that curcumin significantly inhibited the protein expression of Hsp90 $\alpha$. This data suggested that the inhibition of Hsp90 may be one of the mechanisms by which curcumin inhibits HCMV and improves the survival rate of the host cells.

Hsp90 is a highly conserved and essential molecular chaperone at the center of a large protein-folding network (16). It is one of the most abundant chaperone proteins in eukaryotic and prokaryotic cells, and is formed by Hsp90 $\alpha$ and Hsp90 $\beta$ monomer polymerization in higher eukaryotic organisms (17-19). Dysregulation of Hsp90 $\alpha$ is closely associated with the occurrence and development of different types of cancer (17). Therefore, targeting Hsp90 is a promising strategy for the development anticancer drugs.

Hsp90 is a leading member of the heat shock protein family and can form complexes with a variety of proteins. Hsp90 is involved in improving cellular stress tolerance (18), regulating protein conformations (19) and in client protein maturation following transduction (20). Accumulating evidence has demonstrated that Hsp90 and its client proteins cannot only alter the micro-environment of a cell through involvement in cell signaling transduction, cell cycle regulation and cell apoptosis (21), but they also promote viral infections of herpes simplex virus 1, Epstein-Barr virus or intestinal virus by being involved in viral penetration (22), DNA polymerase positioning (14), expression of key proteins (23) and DNA injury repair (24). In agreement with these observations, it was previously revealed that curcumin inhibits the proliferation of HCMV and increases the survival rate of host cells by inhibiting HCMV DNA replication, apoptosis and inflammation (10). The present study used molecular docking simulation analysis to demonstrate that curcumin fit well into the binding pocket of Hsp90. Notably, curcumin eliminated the induction of Hsp90 $\alpha$ 


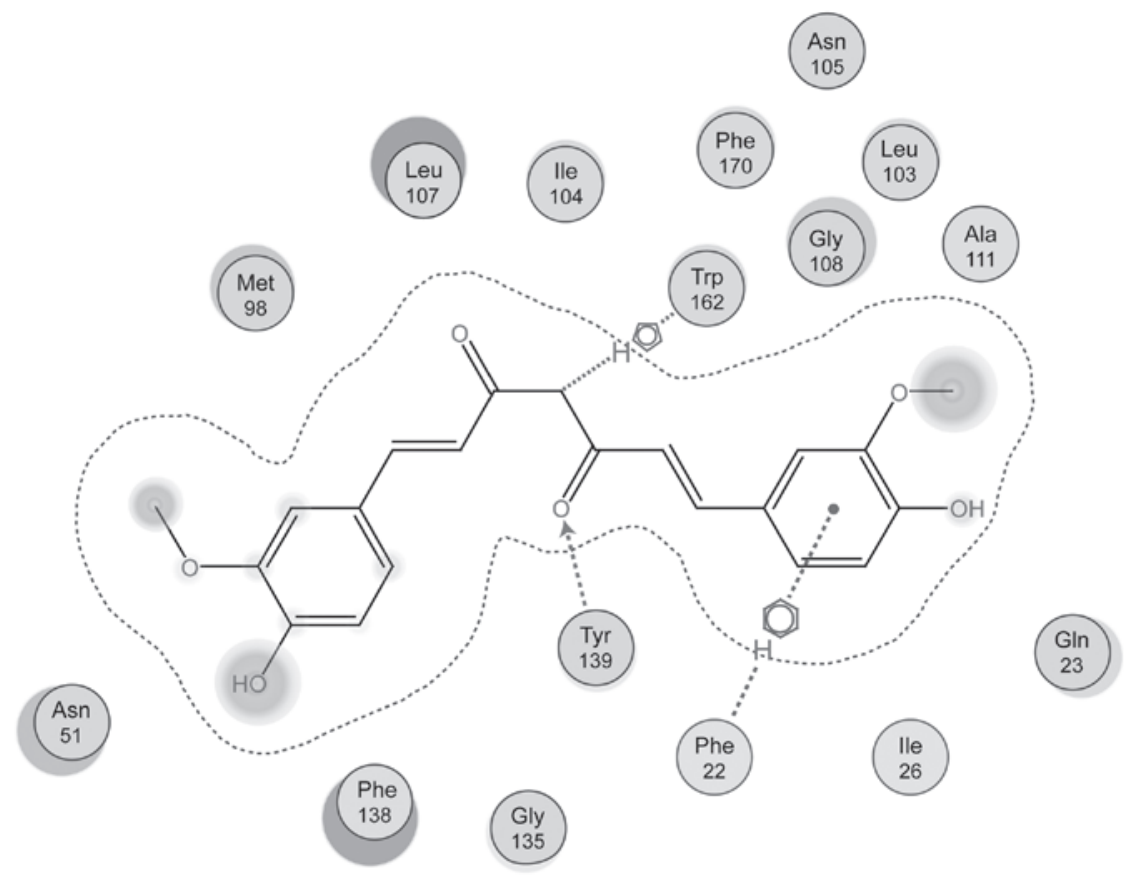

Figure 2. Two-dimensional representation of curcumin binding to heat shock protein 90. A carbonyl oxygen atom of curcumin functions as a hydrogen bond acceptor to form a hydrogen bond with Tyr139. Trp162 and Phe22 and are conjugated to curcumin. In addition, Ile26, Leu107, Ile104, Phe170, Leu103, Ala111 and other hydrophobic amino acids can form hydrophobic interactions with curcumin.
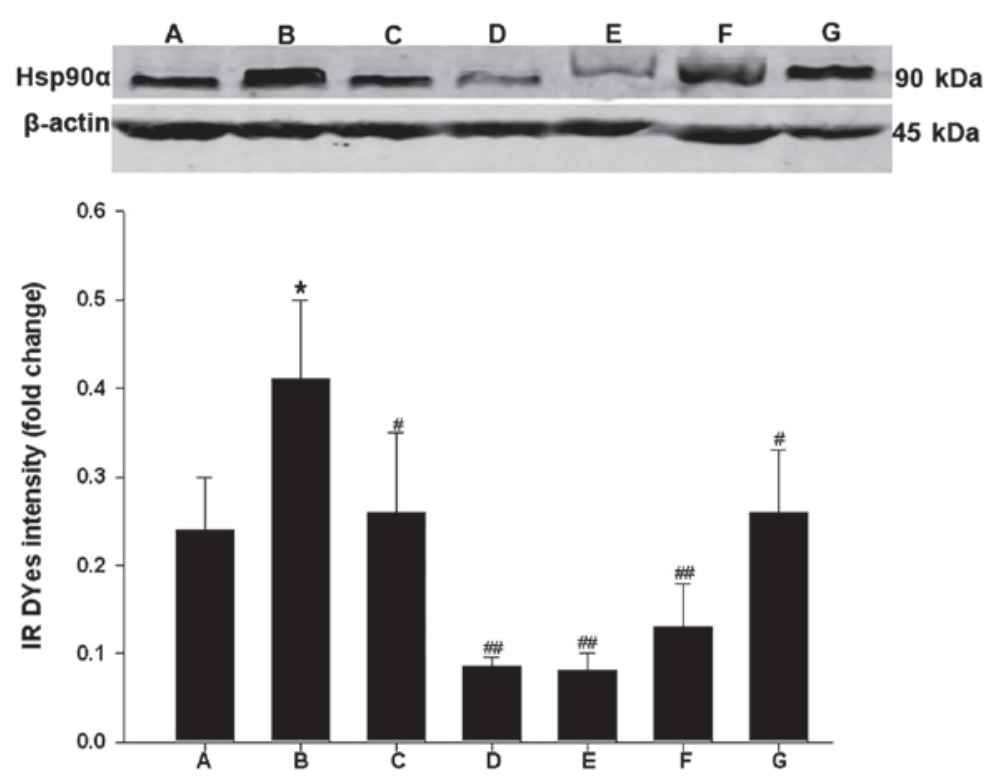

Figure 3. Curcumin decreases the protein expression of Hsp90 $\alpha$. The HELF cells were treatment groups were as follows: (A) HELF cells (control, mock treated); (B) HELF cells + HCMV (model); (C) HELF cells + HCMV + GCV; (D) HELF cells + HCMV + geldanamycin; (E) HELF cells + HCMV + curcumin (high dose, $0.8 \mu \mathrm{g} / \mathrm{ml}$ ); (F) HELF cells + HCMV + curcumin (middle dose, $0.4 \mu \mathrm{g} / \mathrm{ml}$ ); (G) HELF cells + HCMV + curcumin (low dose, $0.2 \mu \mathrm{g} / \mathrm{ml}$ ). The protein expression of $\mathrm{Hsp} 90 \alpha$ was determined using western blotting, and relative protein expression levels were normalized against $\beta$-actin and expressed as the fold change. ${ }^{*} \mathrm{P}<0.05$, compared with the control; ${ }^{\# \#} \mathrm{P}<0.01$ and ${ }^{\#} \mathrm{P}<0.05$, compared with HCMV infection. Data are expressed as the mean \pm standard error of the mean. HELF, Human embryonic lung fibroblast; HCMV, human cytomegalovirus; Hsp $90 \alpha$, heat shock protein $90 \alpha$.

by HCMV infection in the HELF cells, indicating that Hsp90 is involved in the process of viral infection (25-27), and targeting Hsp90 offers a novel promising strategy for the prevention and treatment of infection with viruses, including HCMV.

In conclusion, the present study indicated that the anti-HCMV activity of curcumin is possibly due to its inhibitory effects on Hsp90 $\alpha$. These results suggested that Hsp90 may be a novel target for the treatment of infectious diseases caused by HCMV.

\section{Acknowledgements}

This study was supported by the Medjaden Academy \& Research Foundation for Young Scientists (grant. no. MJR20140019) and 
the Beijing Natural Science Foundation (grant. nos. 7151004 and 7153165). The authors would like to thank Medjaden Bioscience Limited (Hong Kong, China) for assisting in the preparation of this manuscript.

\section{References}

1. Weisblum Y, Panet A, Zakay-Rones Z, Haimov-Kochman R, Goldman-Wohl D, Ariel I, Falk H, Natanson-Yaron S, Goldberg MD, Gilad R, Lurain NS, Greenfield C, Yagel S and Wolf DG. Modeling of human cytomegalovirus maternal-fetal transmission in a novel decidual organ culture. J Virol 85: 13204-13213, 2011.

2. Reuter JD, Gomez DL, Wilson JH and Van Den Pol AN: Systemic immune deficiency necessary for cytomegalovirus invasion of the mature brain. J Virol 78: 1473-1487, 2004.

3. Imai Y, Shum C, Martin DF, Kuppermann BD, Drew WL and Margolis TP: Emergence of drug-resistant cytomegalovirus retinitis in the contralateral eyes of patients with AIDS treated with ganciclovir. J Infect Dis 189: 611-615, 2004.

4. Li S, Zhu J, Zhang W, Chen Y, Zhang K, Popescu LM, Ma X, Lau WB, Rong R, Yu X, et al: Signature microRNA expression profile of essential hypertension and its novel link to human cytomegalovirus infection. Circulation 124: 175-184, 2011.

5. Huang ZR, Yu LP, Yang XC, Zhang F, Chen YR, Feng F, Qian XS and Cai J: Human cytomegalovirus linked to stroke in a Chinese population. CNS Neurosci Ther 18: 457-460, 2012.

6. Shankar TN, Shantha NV, Ramesh HP, Murthy IA and Murthy VS: Toxicity studies on turmeric (Curcuma longa) Acute toxicity studies in rats, guineapigs \& monkeys. Indian J Exp Biol 18: 73-75, 1980.

7. Vallee F, Carrez C, Pilorge F, Dupuy A, Parent A, Bertin L, Thompson F, Ferrari P, Fassy F, Lamberton A, Thomas A, et al: Tricyclic series of heat shock protein 90 (Hsp90) inhibitors part I: Discovery of tricyclic imidazo [4,5-c] pyridines as potent inhibitors of the Hsp90 molecular chaperone. J Med Chem 54: 7206-7219, 2011.

8. Stolina M, Sharma S, Lin Y, Dohadwala M, Gardner B, Luo J, Zhu L, Kronenberg M, Miller PW, Portanova J, et al: Specific inhibition of cyclooxygenase 2 restores antitumor reactivity by altering the balance of IL-10 and IL-12 synthesis. J Immunol 164 361-370, 2000.

9. Pfaffl MW: A new mathematical model for relative quantification in real-time RT-PCR. Nucleic Acids Res 29: e45, 2001.

10. Lv YL, Lan AJ, Lan YY, Li C, Lei N, Wu C and Liu LH: Activity of curcumin against human cytomegalovirus in vitro. Afr J Pharm Pharmacol 6: 30-35, 2012.

11. Austen $M$ and Dohrmann C: Phenotype-first screening for the identification of novel drug targets. Drug Discov Today 10 275-282, 2005.

12. Lu XC, Chi XH, Yang B, Zhu HL, Liu LH, Zhang F and Yan JW: Gene expression profile analysis of $\mathrm{T}$ lymphocytes involved in pathogenesis of severe aplastic anemia by using bioinformatics method as a novel way of drug screening. Zhongguo Shi Yan Xue Ye Xue Za Zhi 18: 416-420, 2010 (In Chinese).
13. Yang B, Lu XC, Liu LH, Zhu HL, Chi XH, Yao SQ, Lou FD and $\mathrm{Yu}$ L: Experimental identification of drugs with function of targeted up-regulating ID4 expression: Bioinformatics-based prediction and preliminary validation. Zhonghua Yi Xue Za Zhi 89: 1714-1716, 2009 (In Chinese).

14. Burch AD and Weller SK: Herpes simplex virus type 1 DNA polymerase requires the mammalian chaperone hsp90 for proper localization to the nuleus. J Virol 79: 10740-10749, 2005.

15. Grenert JP, Sullivan WP, Fadden P, Haystead TA, Clark J, Mimnaugh E, Krutzsch H, Ochel HJ, Schulte TW, Sausville E, et al: The amino-terminal domain of heat shock protein 90 (hsp90) that binds geldanamycin is an ATP/ADP switch domain that regulates hsp90 conformation. J Biol Chem 272: 23843-23850, 1997.

16. Geller R, Andino R and Frydman J: Hsp90 inhibitors exhibit resistance-free antiviral activity against respiratory syncytial virus. PLoS One 8: e56762, 2013.

17. Cheng CF, Fan J, Zhao ZW, Woodley DT and Li W: Secreted heat shock protein-90a: A more effective and safer target and for anti-cancer drags. Curr Signal Tranduction Ther 5: 121-127, 2010.

18. Zhai QQ, Luo Y, Wang QD, Xia M, Xing GW, Li YC, Jiang JH, Liu Z, Liu QY, Wang YF, et al: Determination of SNX-2112, a selective Hsp90 inhibitor, in plasma samples by hish-performance liquid chromatography and its application to pharmacokineties in rats. J Pharm Biomed Anal 53: 1048-1052, 2010.

19. Jiang S, QL, Zhou F, Huang J, Guo Y and Yang K: Molecular cloning and expression analysis of a heat shock protein (Hsp90) gene from black tiger shrimp (Penaeus monodon). Mol Biol Rep 36: 127-134, 2009.

20. Meng S SG and Li ZR: Recent progress in the study of Hsp90 inhibitors. Chin J Antibiot 36: 241-248, 2011.

21. Wen KW and Damania B: Hsp90 and Hsp40/Erdj3 are required for the expression and anti-apoptotic function of KSHV K1. Oncogene 29: 3532-3544, 2010.

22. Tsou YL, Lin YW, Chang HW, Lin HY, Shao HY, Yu SL, Liu CC, Chitra E, Sia C and Chow YH: Heat shock protein 90: Role in enterovirus 71 entry and assembly and potential target for therapy. PLoS One 8: e77133, 2013.

23. Murata T, Iwata S, Siddiquey MN, Kanazawa T, Goshima F, Kawashima D, Kimura H and Tsurumi T: Heat shock protein 90 inhibitors repress latent membrane protein 1 (LMP1) expression and proliferation of Epstein-Barr virus-positive natural killer cell lymphoma. PLoS One 8: e63566, 2013.

24. Luo Y, Lou S, Deng X, Liu Z, Li Y, Kleiboeker S and Qiu J: Parvovirus B19 infection of human primary erythroid progenitor cells triggers ATR-Chk1 signaling, which promotes B19 virus replication. J Virol 85: 8046-8055, 2011.

25. Geller R, Vignuzzi M, Andino R and Frydman J: Evolutionary constraints on chaperone-mediated folding provide an antiviral approach refractory to development of drug resistance. Genes Dev 21: 195-205, 2007.

26. Turnell AS and Grand RJ: DNA viruses and the cellular DNA-damage response. J Gen Virol 93: 2076-2097, 2012.

27. Xiao A, Wong J and Luo H: Viral interaction with molecular chaperones: Role in regulating viral infection. Arch Virol 155: 1021-1031, 2010 